\title{
Single top production and properties at hadron colliders
}

\section{Kirill Skovpen*}

on behalf of the ATLAS, CDF, CMS and DO Collaborations, Institut Pluridisciplinaire Hubert Curien, CNRS/IN2P3, Strasbourg, France

E-mail: Kirill.Skovpen@cern.ch

The results of cross section measurements for electroweak production of top quarks in t channel, s channel, and in association with W-boson are presented from LHC and Tevatron experiments. These measurements are used to place constraints on the CKM matrix element $V_{t b}$. Top quark polarization is studied in the measurement of the top quark spin asymmetry in single top events. Anomalous structure of top quark coupling is probed.

Fourth Annual Large Hadron Collider Physics

13-18 June 2016

Lund, Sweden

*Speaker. 


\section{Introduction}

Processes of single top quark production provide unique environment to directly probe electroweak interactions at hadron colliders. Wtb vertex is involved in all single top production mechanisms and allows one to determine the CKM matrix element $V_{t b}$ directly from the measured cross sections, as well as to probe parton distribution functions (PDF) in proton. New physics processes related to anomalous Wtb coupling structure, as well as flavor-changing neutral currents (FCNC) with top quarks, can be looked for in single top events. This report summarizes the latest results on the single top quark processes from Tevatron [1,2] and LHC [3, 4] experiments.

\section{Single top production}

In the Standard Model (SM) there are three main single top production mechanisms: $t$ channel, $\mathrm{s}$ channel and $\mathrm{Wt}$ channel. In case of the $\mathrm{t}$ channel, there is a light quark recoiling from a t $\mathrm{b}$ system. In $\mathrm{s}$ channel the top quark is produced together with a $\mathrm{b}$ quark via a virtual W-boson. And, finally, in the Wt channel the top quark is produced in association with a W-boson. The dominant single top production mode at hadron colliders is $t$ channel. Cross section for $\mathrm{s}$ channel is roughly two times smaller than that of $\mathrm{t}$ channel at Tevatron, while at LHC its cross section is the smallest one among all the three production mechanisms. Predicted cross sections for single top processes are presented in Tab. 1. Cross sections are calculated at NNLO while the Monte-Carlo (MC) generation of events is done at NLO with NLO+NNLL generation available for Wt channel.

Table 1: Predicted cross sections for single top quark production processes.

\begin{tabular}{cccc}
\hline Energy & Process & Cross section [pb] & Reference \\
\hline \multirow{2}{*}{ Tevatron $(1.96 \mathrm{TeV})$} & $\mathrm{t}$ & $2.10 \pm 0.13$ & {$[5]$} \\
& $\mathrm{s}$ & $1.05 \pm 0.06$ & {$[5]$} \\
& $\mathrm{Wt}$ & $0.25 \pm 0.03$ & {$[6]$} \\
\hline LHC $(7 \mathrm{TeV})$ & $\mathrm{t}$ & $65.9_{-0.7}^{+2.1}(\text { scale })_{-1.7}^{+1.5}(\mathrm{PDF})$ & \\
& $\mathrm{s}$ & $4.56 \pm 0.07(\text { scale })_{-0.17}^{+0.18}(\mathrm{PDF})$ & {$[7]$} \\
& $\mathrm{Wt}$ & $15.6 \pm 0.4($ scale $) \pm 1.1(\mathrm{PDF})$ & \\
\hline & $\mathrm{t}$ & $87.2_{-1.0}^{+2.8}(\text { scale })_{-2.2}^{+2.0}(\mathrm{PDF})$ & \\
LHC $(8 \mathrm{TeV})$ & $\mathrm{s}$ & $5.55 \pm 0.08($ scale $) \pm 0.21(\mathrm{PDF})$ & {$[7]$} \\
& $\mathrm{Wt}$ & $22.2 \pm 0.6($ scale $) \pm 1.4(\mathrm{PDF})$ & \\
\hline & $\mathrm{t}$ & $216.99_{-4.64}^{+6.62}($ scale $) \pm 6.16(\mathrm{PDF})$ & {$[8]$} \\
LHC $(13 \mathrm{TeV})$ & $\mathrm{s}$ & $10.3 \pm 0.4$ & {$[9]$} \\
& $\mathrm{Wt}$ & $71.1 \pm 3.8$ & {$[9]$} \\
\hline
\end{tabular}

\section{Single top production at Tevatron}

The latest results from Tevatron with the total integrated luminosity of up to $9.7 \mathrm{fb}^{-1}$ represent a combination of the results from D0 and CDF experiments for $t$ and $s+t$ channels [10]. This result 
complements already reported s-channel measurement [11]. This analysis represents a statistically independent combination of the results obtained in lepton+jets and $E_{T}^{\text {miss }}+$ jets channels. To select signal events a Multivariate analysis (MVA) approach is used where the final discriminant is defined as a signal over background ratio calculated in bins of MVA output, with the signal defined as a difference between $t$ and $s$ channel events. Such definition allows one to have a distinctive separation between $t$ and $s$ channel events in one-dimensional representation. The resulting twodimensional posterior probability distribution as a function of the measured $t$ and $s$ channel cross sections is presented in Fig. 1, where the predicted cross section along with several beyond the SM (BSM) predictions are shown. The posterior probability distribution as a function of $\left|V_{t b}\right|^{2}$ is shown in Fig. 1. The measured cross sections and $\left|V_{t b}\right|$ are consistent with the SM predictions. Fig. 2 summarizes all measurements done at Tevatron for single top production processes.
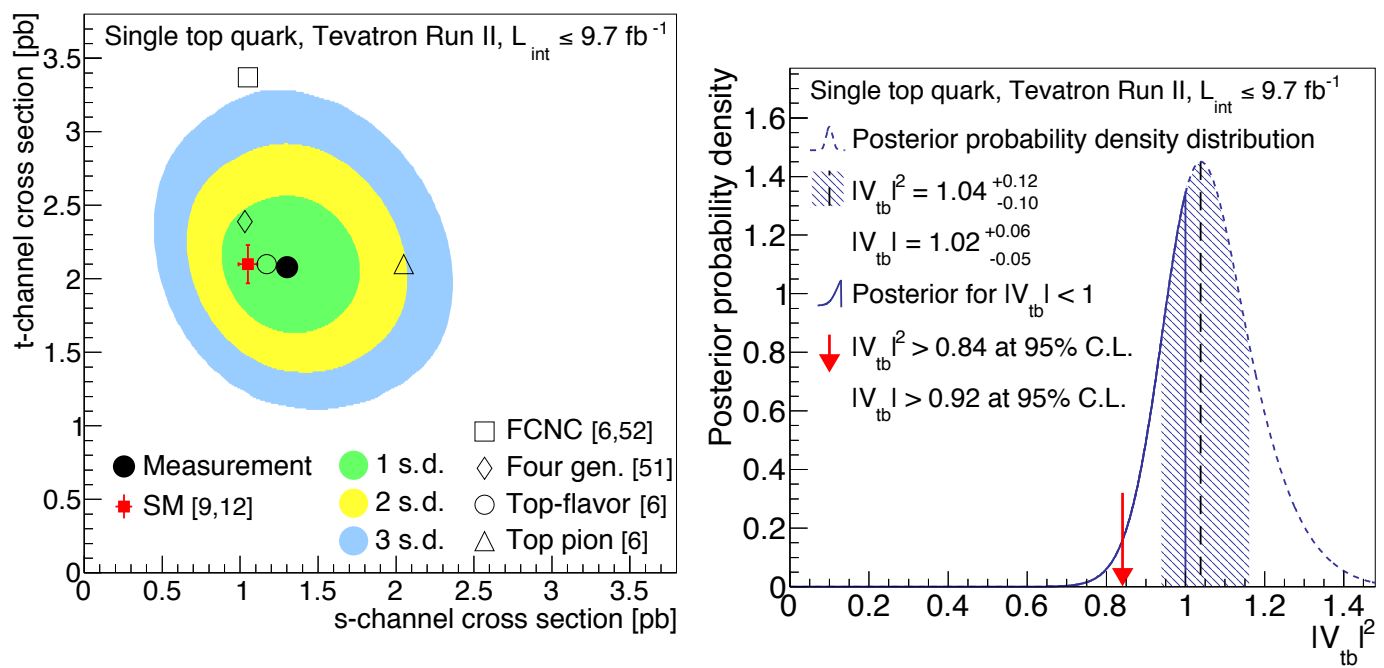

Figure 1: Two-dimensional posterior probability as a function of $\mathrm{t}$ and $\mathrm{s}$ channel cross sections (left) and posterior probability distribution as a function of $\left|V_{t b}\right|^{2}$ (right) [11].

\section{Single top production at $\mathrm{LHC}$}

\section{1 t-channel}

A measurement of $t$ channel production cross section with $2.3 \mathrm{fb}^{-1}$ at CMS [12] represents an update on the first $t$ channel measurement done with the early data of $42 \mathrm{pb}^{-1}$ at $13 \mathrm{TeV}$ [13]. The measurement is done in muon+jets channel. In addition to the signal region with two reconstructed jets, one of which is required to be $b$ tagged, there are several control regions defined based on the number of reconstructed non- $b$ and $b$ tagged jets in order to simultaneously constrain all the important backgrounds through a combined binned likelihood fit a Neural network discriminant which is shown in Fig. 3. t channel cross section measurements at different center-of-mass energies is presented in Fig. 4, where the measured cross sections show very good agreement with predicted values. The extracted value for $t$ channel cross section is $227.8 \pm 9.1$ (stat.) \pm 14.0 (exp.) ${ }_{-27.7}^{+28.7}$ (theo.) \pm 6.2 (lumi.) pb. The measured value of $\left|V_{t b}\right|$ is $1.02 \pm 0.07$ (exp.) \pm 0.02 (theo.). 


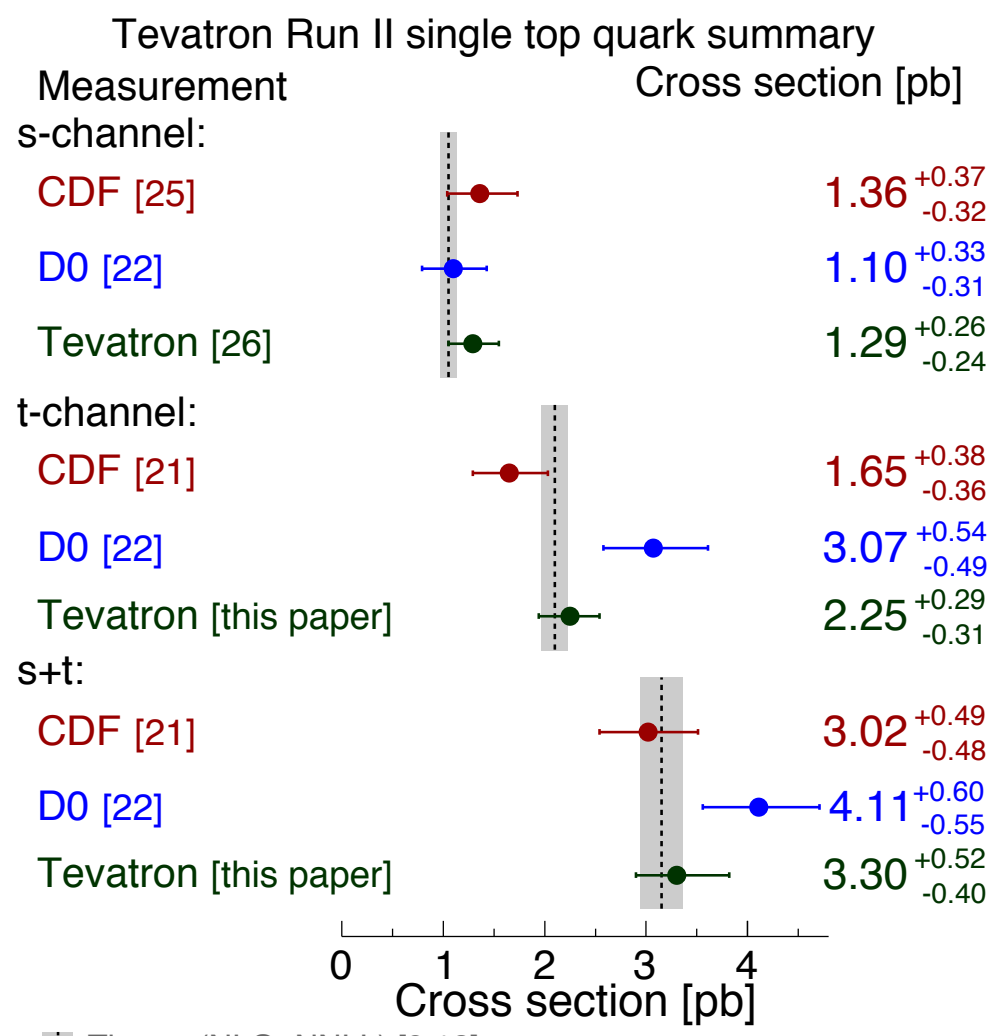

Theory (NLO+NNLL) $[9,12]$

$$
\mathrm{m}_{\mathrm{t}}=172.5 \mathrm{GeV}
$$

Figure 2: Measured cross sections for single top production processes at Tevatron [10].

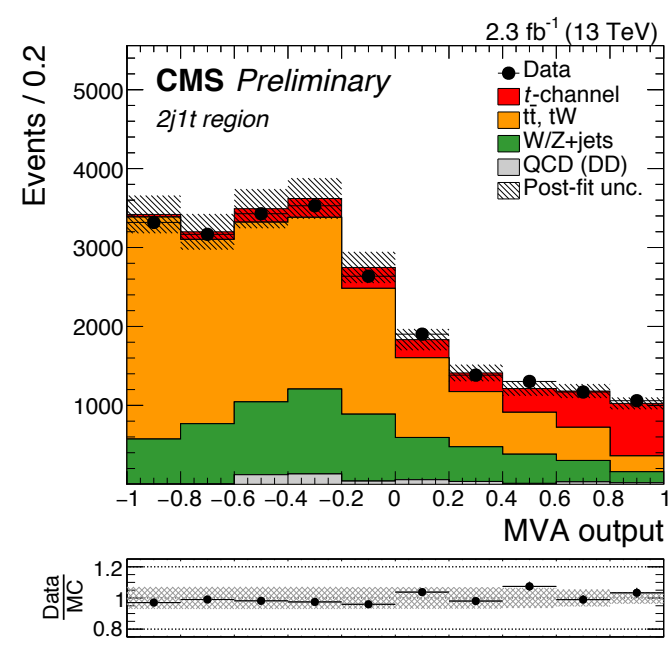

Figure 3: Neural network distribution normalized to the yields obtained from the simultaneous fit [12]. 


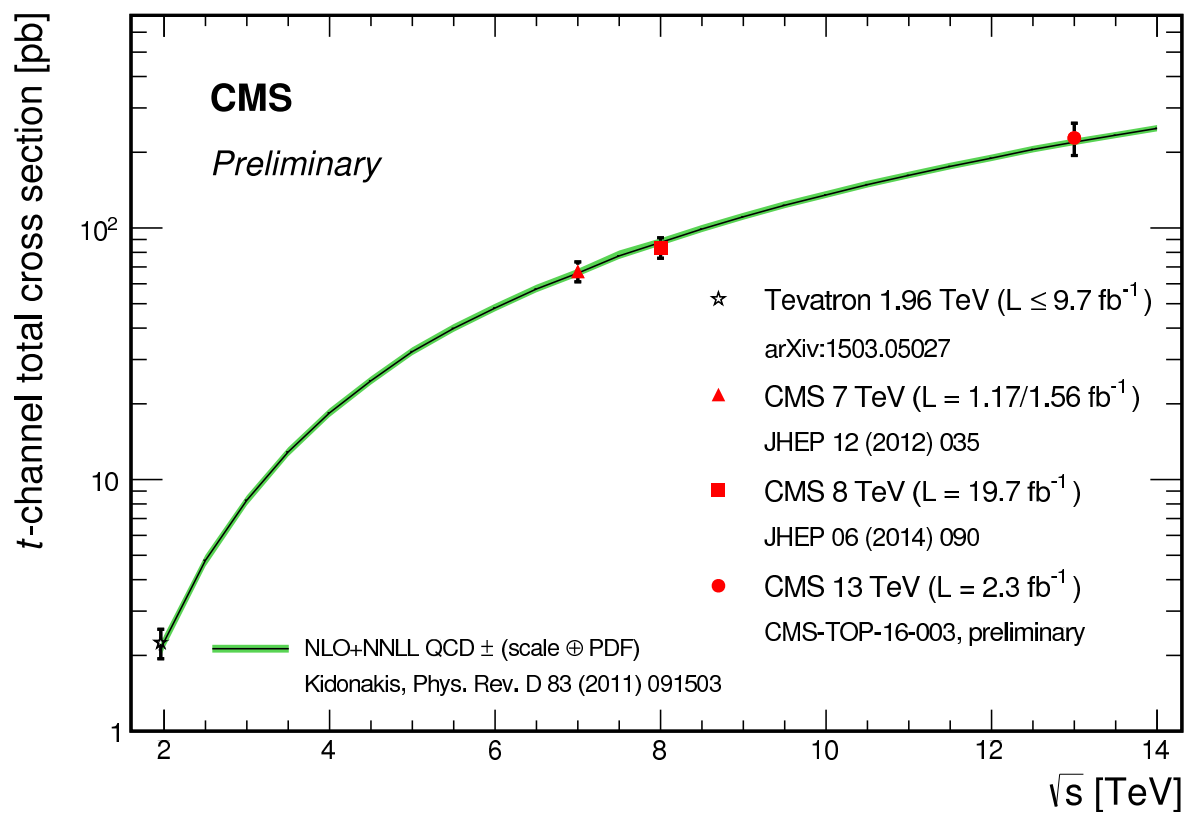

Figure 4: The t channel single top cross section summary in comparison with theoretical predictions [12].

The $t$ channel cross section measurement performed separately for top and antitop quarks at $13 \mathrm{TeV}$ is done at ATLAS [14]. The analysis strategy and event selection criteria are quite similar to the ones considered at CMS, with the measurement done in muon+jets channel and all the dominant backgrounds constrained through a likelihood fit. One of the most important kinematic variables used in Neural network is the transverse mass of the reconstructed W-boson $\left(m_{T}(W)\right)$. The distributions for this variable after the final selection criteria applied and the Neural network discriminant are shown in Fig. 5. The measured cross section for single production of top and antitop quark via $t$ channel is $133 \pm 6$ (stat.) \pm 24 (syst.) \pm 7 (lumi.) pb and $96 \pm 5$ (stat.) \pm 23 (syst.) \pm 5 (lumi.) $\mathrm{pb}$, respectively. The extracted value for $\left|V_{t b}\right|$ is $1.03 \pm 0.02$ (stat.) \pm 0.11 (syst.) \pm 0.02 (theo.) \pm 0.03 (lumi.).

The first $t$ channel differential cross section measurement is done at CMS with $13 \mathrm{TeV}$ data [15]. Signal events are extracted from a binned likelihood fit to a Boosted Decision Tree (BDT) discriminator after the selection of $m_{T}(W)>50 \mathrm{GeV}$, as well as to $m_{T}(W)$ for $m_{T}(W)<50 \mathrm{GeV}$, in the signal and background control regions. The cross section is measured as a function of the top quark $p_{T}$ and rapidity after the unfolding procedure applied to the results obtained at reconstruction level. Results are compared to different generators in Fig. 6 for different flavor schemes and hadronization configurations. Comparisons show fairly good agreement.

\section{2 s-channel}

Observation of the s channel at LHC is very challenging because of the smallness of the production cross section. Based on the full $8 \mathrm{TeV}$ data analysis evidence for this production channel was reported by ATLAS [16]. The significance of the reported result is $3.2 \sigma$ with $3.9 \sigma$ expected. This is an update on the measurement with the observed significance of $1.3 \sigma$ for this 

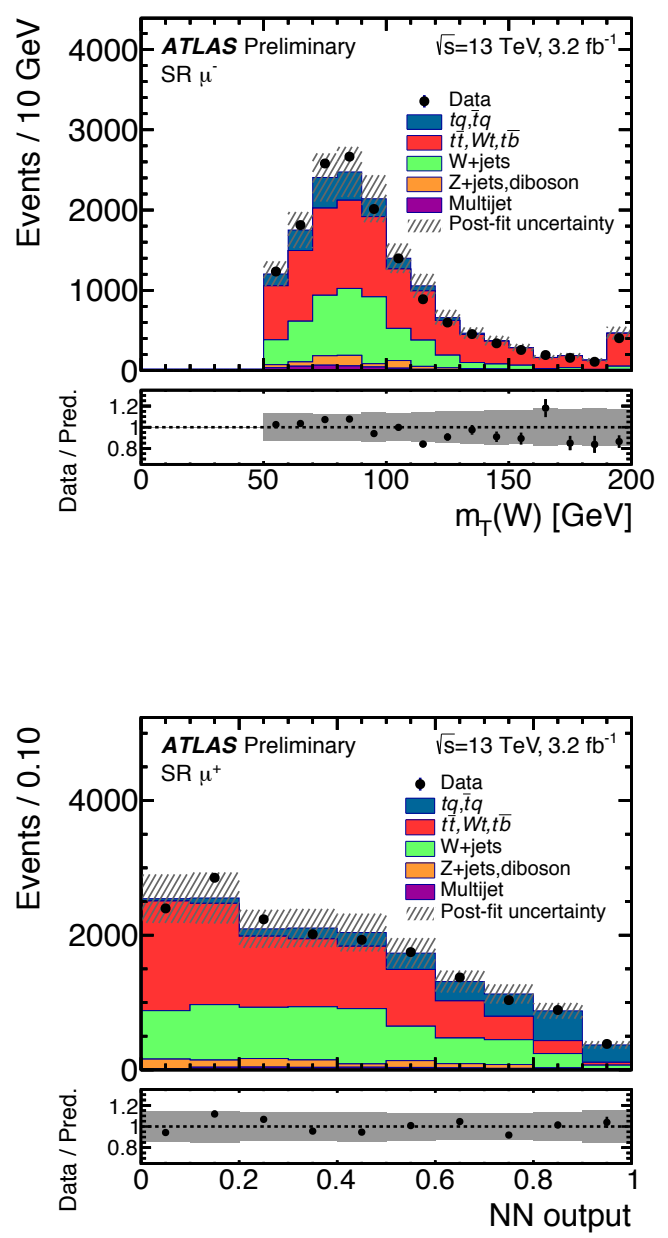

Figure 5: Distribution for transverse mass of the reconstructed W-boson (top) and Neural network discriminator (bottom) [14].

channel previously done at ATLAS [17] that used BDT approach to define the final discriminant. The new analysis uses the same dataset but with a different analysis approach of Matrix element (ME) method to select signal events. The application of this method contributed to roughly $50 \%$ improvement in the expected significance with respect to BDT-based analysis, with the other 50\% of improvement from the use of new MC samples with increased statistics, as well as the latest detector calibrations and further optimized analysis selection criteria. Signal events are extracted through a simultaneous binned likelihood fit to a ME discriminant and a lepton charge distribution in signal and background control regions. The comparison of simulated s channel top quark events and data with background subtracted is presented in Fig. 7. The measured cross section for s channel process is $4.8 \pm 0.8$ (stat.) ${ }_{-1.3}^{+1.6}$ (syst.) pb. Lower limit of 0.5 on $\left|V_{t b}\right|$ at $95 \%$ CL is set.

The s channel process has also been looked for at CMS [18]. A combined fit to 7 and $8 \mathrm{TeV}$ 

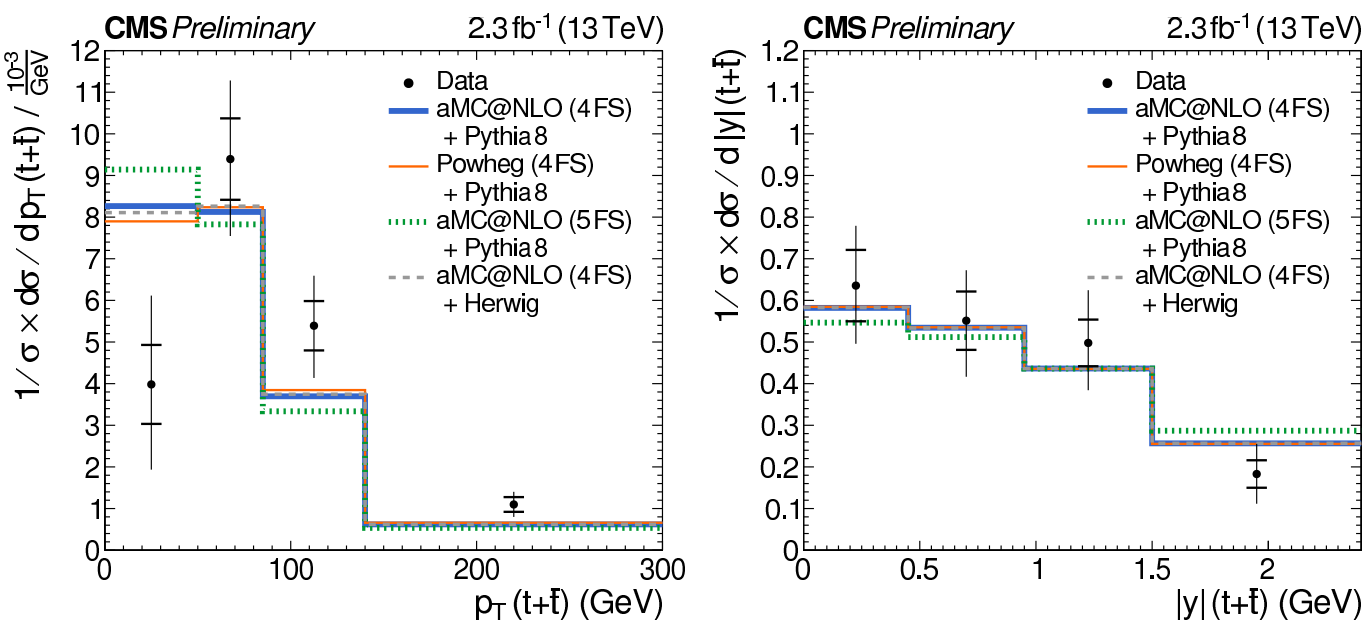

Figure 6: Measured differential cross section of t channel single top production as a function of top quark $p_{T}$ (left) and rapidity (right) [15].

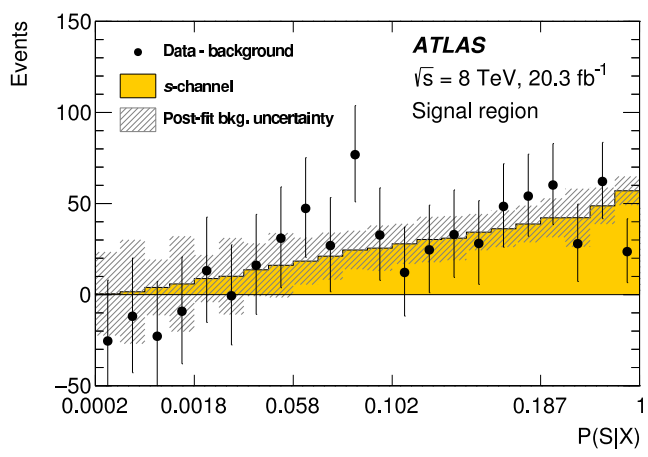

Figure 7: Distribution of the ME discriminant in data in the signal region after the subtraction of all backgrounds compared to the prediction [16].

data results is performed to determine the signal cross section relative to SM predictions. In $7 \mathrm{TeV}$ analysis the muon+jets channel was studied, while at $8 \mathrm{TeV}$ both electron and muon channels were combined. The analysis strategy based on BDT approach is to define the discriminant to select signal events with all the dominant backgrounds constrained through a simultaneous likelihood fit. Analyzes at 7 and $8 \mathrm{TeV}$ yield cross sections of $7.1 \pm 8.1 \mathrm{pb}$ and $13.4 \pm 7.3 \mathrm{pb}$, respectively, with a best fit value of $2.0 \pm 0.9$ for the combined ratio of the measured and expected values. The observed significance of the combined measurement is $2.5 \sigma$ with $1.1 \sigma$ expected.

\subsection{Wt channel}

Production of a single top quark with a W-boson is second dominant process at LHC in terms of the predicted cross section. The first observation of this channel was reported by CMS at 8 $\mathrm{TeV}$ [19]. The analysis was done in the final state with two oppositely charged leptons. Backgrounds are suppressed with a BDT approach and the signal is extracted from a binned likelihood 
fit using the BDT discriminant. The measured cross section is $23.4 \pm 5.4 \mathrm{pb}$ with the observed significance of $6.1 \sigma$ which means the observation of this process. The lower limit on $\left|V_{t b}\right|$ of 0.78 at $95 \% \mathrm{CL}$ is obtained with the measured value of $1.03 \pm 0.12$ (exp.) \pm 0.04 (theo.).

The Wt channel was also observed at ATLAS at $8 \mathrm{TeV}$ [20]. Similarly to CMS, a BDT approach was considered to select signal events with the signal extraction proceeded through a profile likelihood fit. The measured cross section is $23.0 \pm 1.3$ (stat.) ${ }_{-3.5}^{+3.2}$ (syst.) \pm 1.1 (lumi.) pb. The observed significance of the measurement is $7.7 \sigma$ with $6.9 \sigma$ expected. In this analysis, in addition to the inclusive cross section, the first fiducial cross section was measured. The fiducial region definition corresponds to the selection of two leptons with $p_{T}>25 \mathrm{GeV}$ and $|\eta|<2.5$, one b-tagged jet with $p_{T}>20 \mathrm{GeV}$ and $|\eta|<2.5$, and $E_{T}^{\text {miss }}>20 \mathrm{GeV}$. The cross section measured in fiducial region allows one to have a more robust comparison to theoretical predictions without an extrapolation to regions outside of the detector acceptance. The measured fiducial cross section of $0.85 \pm 0.01$ (stat.) ${ }_{-0.07}^{+0.06}$ (syst.) \pm 0.03 (lumi.) $\mathrm{pb}$ is compared to various generators with different PDFs and hadronization configurations in Fig. 8. To account for interference between Wt channel and top quark pair production different techniques are used, such as diagram removal and diagram subtraction. The extracted value for $\left|V_{t b}\right|$ is $1.01 \pm 0.10$ with the lower limit of 0.80 at $95 \% \mathrm{CL}$.

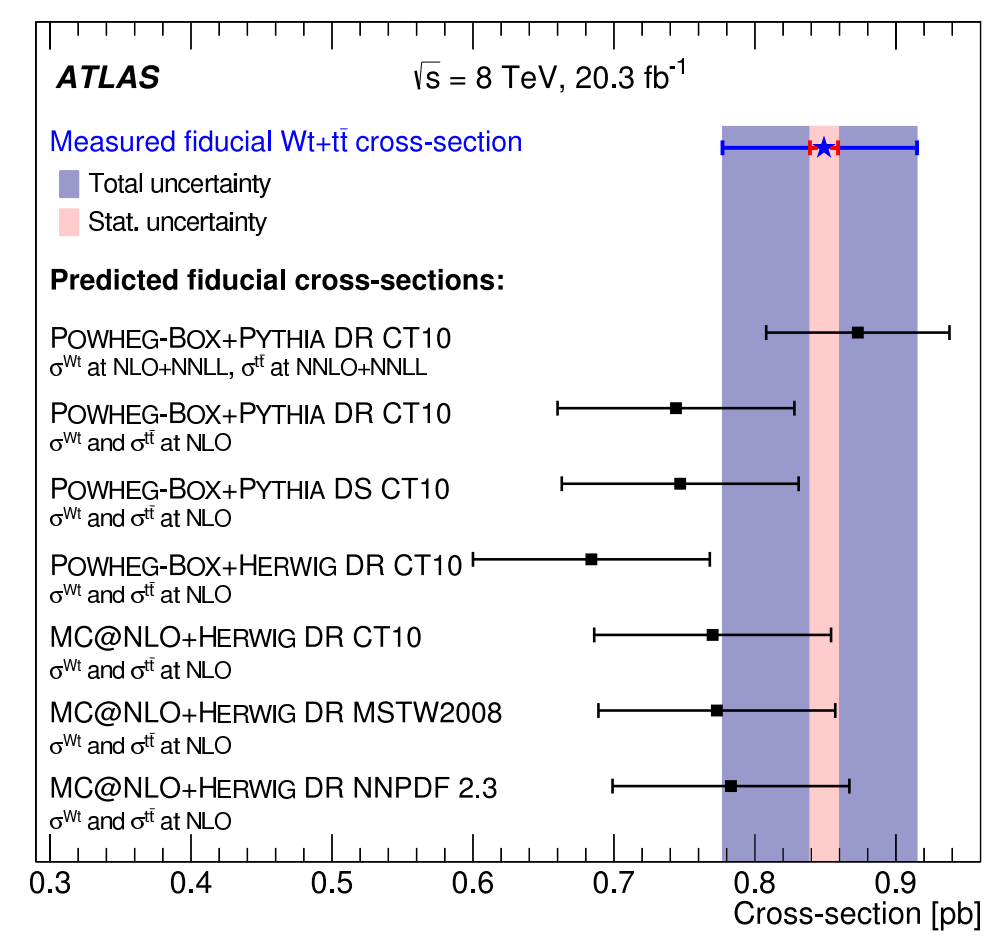

Figure 8: Comparison of the measured fiducial cross section to theoretical predictions [20].

A dedicated effort for a combination of the results for Wt channel cross section from ATLAS and CMS at $8 \mathrm{TeV}$ yields a cross section of $23.1 \pm 1.1$ (stat.) \pm 3.3 (syst.) \pm 0.8 (lumi.) pb and $\left|V_{t b}\right|$ value of $1.02 \pm 0.09$ [21,22]. The combination is performed with the best linear unbiased estimator (the BLUE method) and gives a slight improvement in the precision of the measurement 
of $16 \%$ for the total uncertainty, mostly driven by the ATLAS result. The results of individual measurements and the final combination are summarized in Fig. 9.

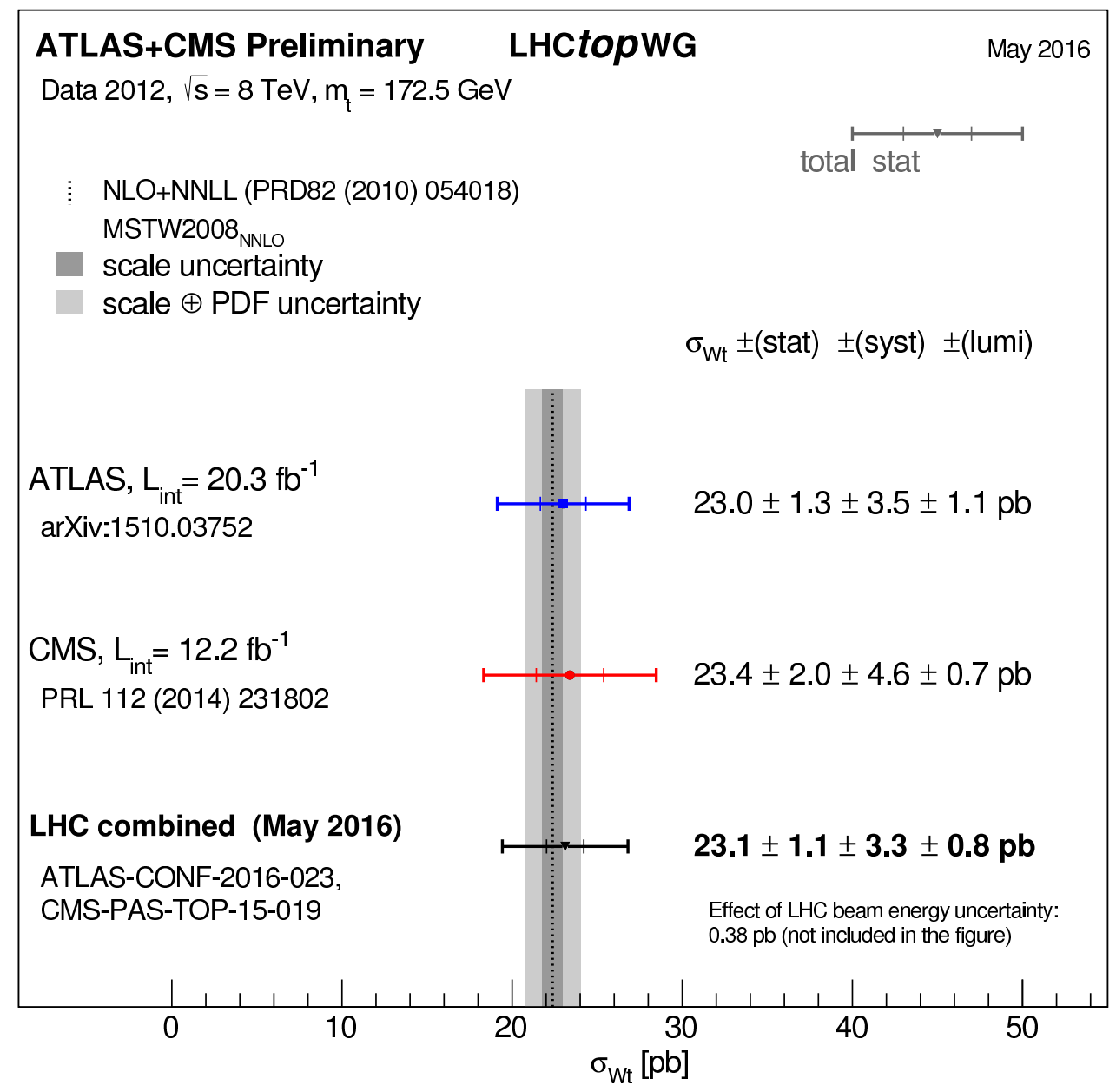

Figure 9: Cross section measurements for Wt channel performed by ATLAS and CMS, and the combined result, compared to theoretical predictions [21,22].

Cross section and $\left|V_{t b}\right|$ results for all single top production channels are presented on Figs. 10 and 11, respectively [23]. Comparisons show that all experimental results are in good agreement with SM predictions.

\section{Top quark properties}

Processes with single top production allow one to probe Wtb anomalous couplings directly in the measured production rates of top quarks, as well as in various kinematic and angular distributions which could be modified in the presence of anomalous interactions. The high polarization of top quarks $(\mathrm{P} \simeq 0.9)$ introduces an additional degree of freedom to study angular asymmetries sensitive to $\mathrm{Wtb}$ vertex structure. 


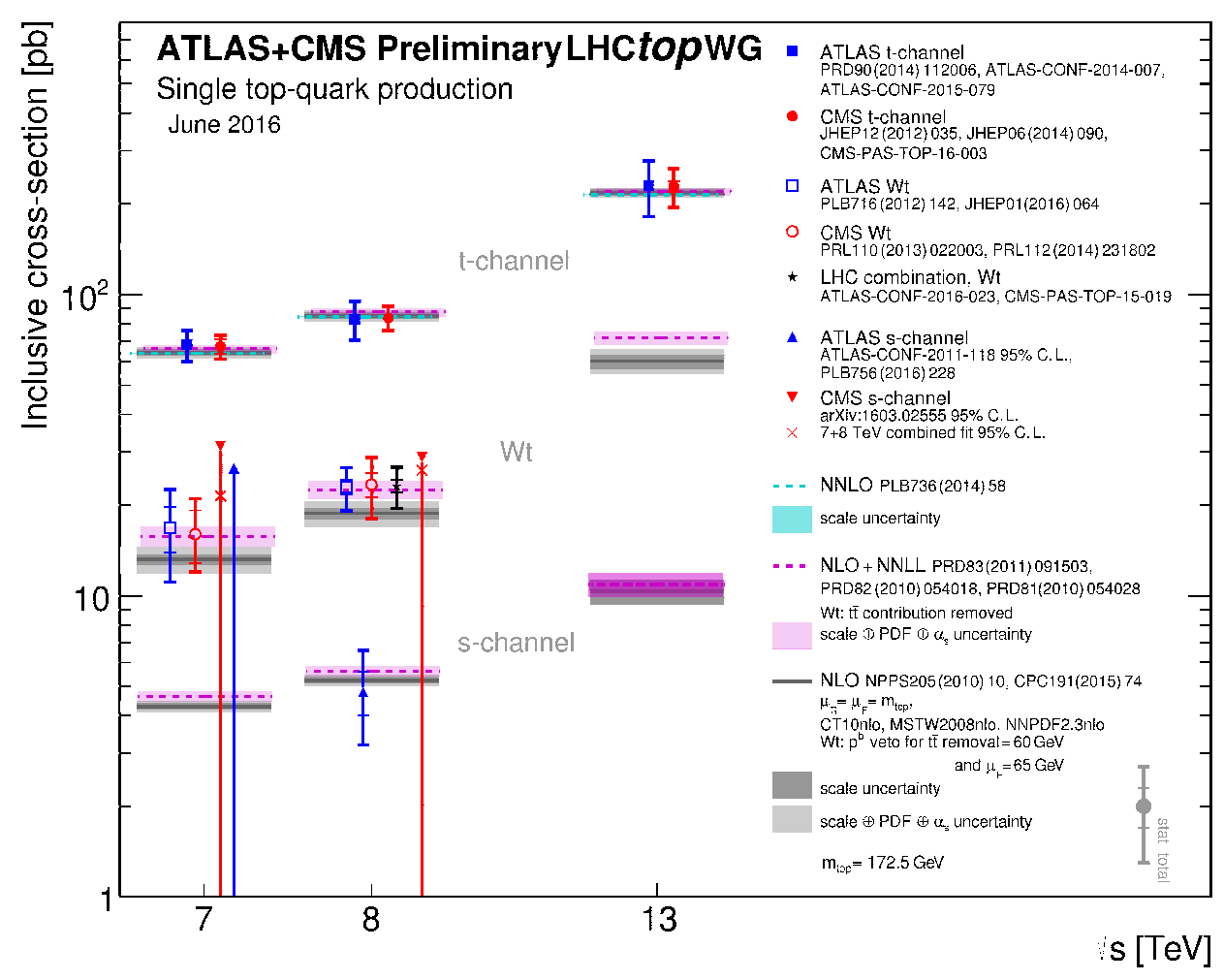

Figure 10: Summary of ATLAS and CMS measurements of the single top production cross sections in various channels as a function of the center-of-mass energy [23].

\subsection{Top quark polarization}

The first measurement of top quark spin asymmetry $\left(A_{\mu}\right)$ in high-purity single top $\mathrm{t}$ channel events was done at CMS with $8 \mathrm{TeV}$ data [24]. Top quark spin projection is measured along the direction of the recoiling spectator quark momentum. One of the kinematic variables to the polarization of the top quark is $\theta^{*}$ which is defined as an angle between top quark spin direction and muon momentum in the top quark rest frame. The cross section for $t$ channel process is measured as a function of the $\cos \theta^{*}$, unfolded at parton level. Then, a fit is done on the unfolded $\cos \theta^{*}$ differential cross section to estimate the asymmetry. The reconstructed distribution for $\cos \theta^{*}$ and the unfolded result for the measured differential cross section are presented in Fig. 12. There is a fairly good agreement between the data and theoretical calculations, although a slight trend might be seen in the level of the agreement. The extracted asymmetries, $A_{\mu}(t)=0.29 \pm 0.11, A_{\mu}(\bar{t})=$ $0.21 \pm 0.14$ and $A_{\mu}(t+\bar{t})=0.26 \pm 0.11$, are in agreement with the SM expectation of $A_{\mu}^{S M}=0.44$ with a p-value of $4.6 \%$.

\subsection{Double differential asymmetry}

A normalized double differential angular measurement with respect to $\theta^{*}$ and $\phi^{*}$ as defined in Fig. 13 is done at ATLAS with $8 \mathrm{TeV}$ data [25]. This measurement is sensitive to both real and imaginary parts of Wtb anomalous couplings, and represents a search for $\mathrm{CP}$ violation. The langrangian for anomalous Wtb interactions can be expressed in the following way: 


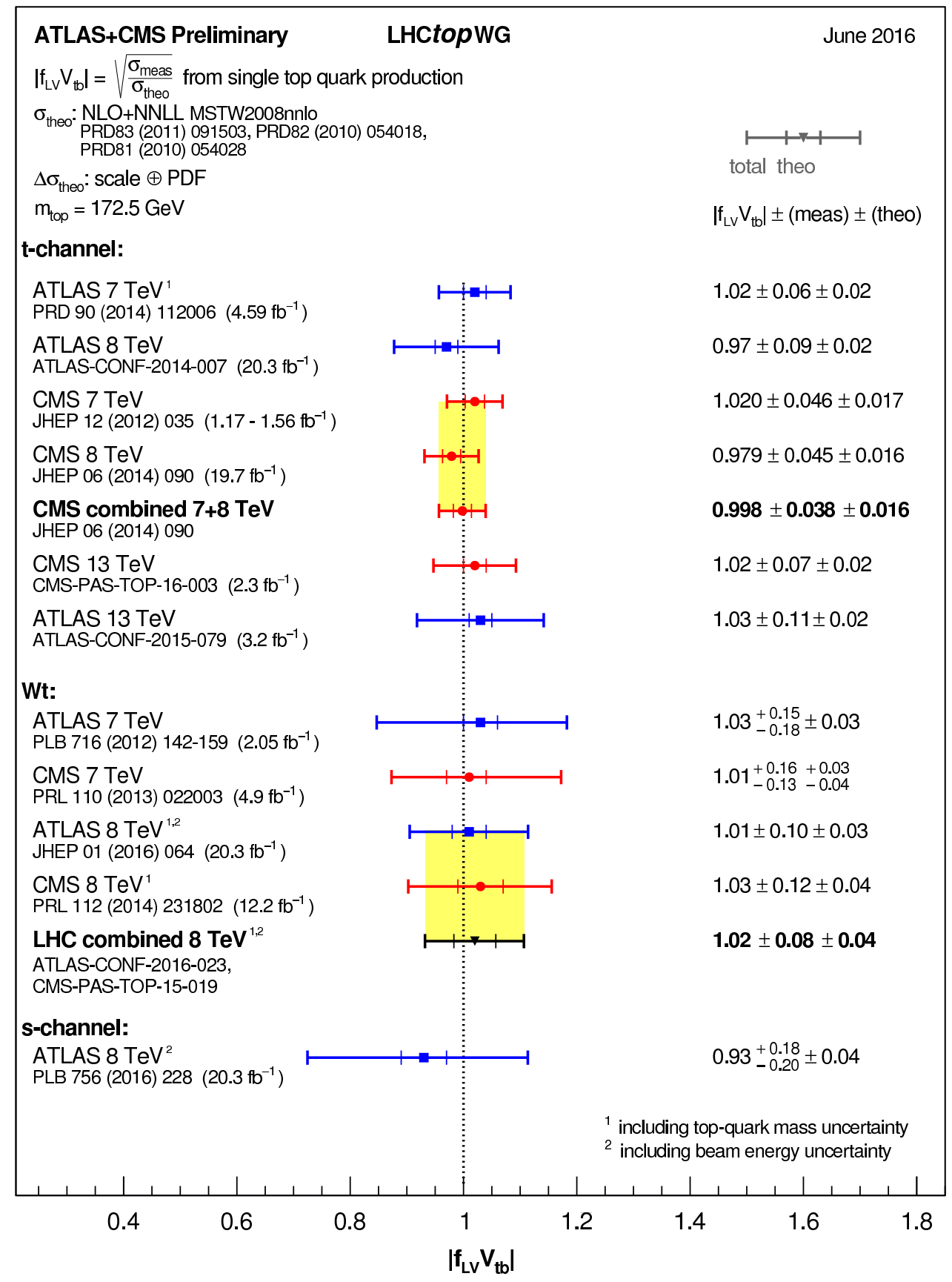

Figure 11: Summary of the ATLAS and CMS extractions of the CKM matrix element $\left|V_{t b}\right|$ from single top quark measurements [23]. 

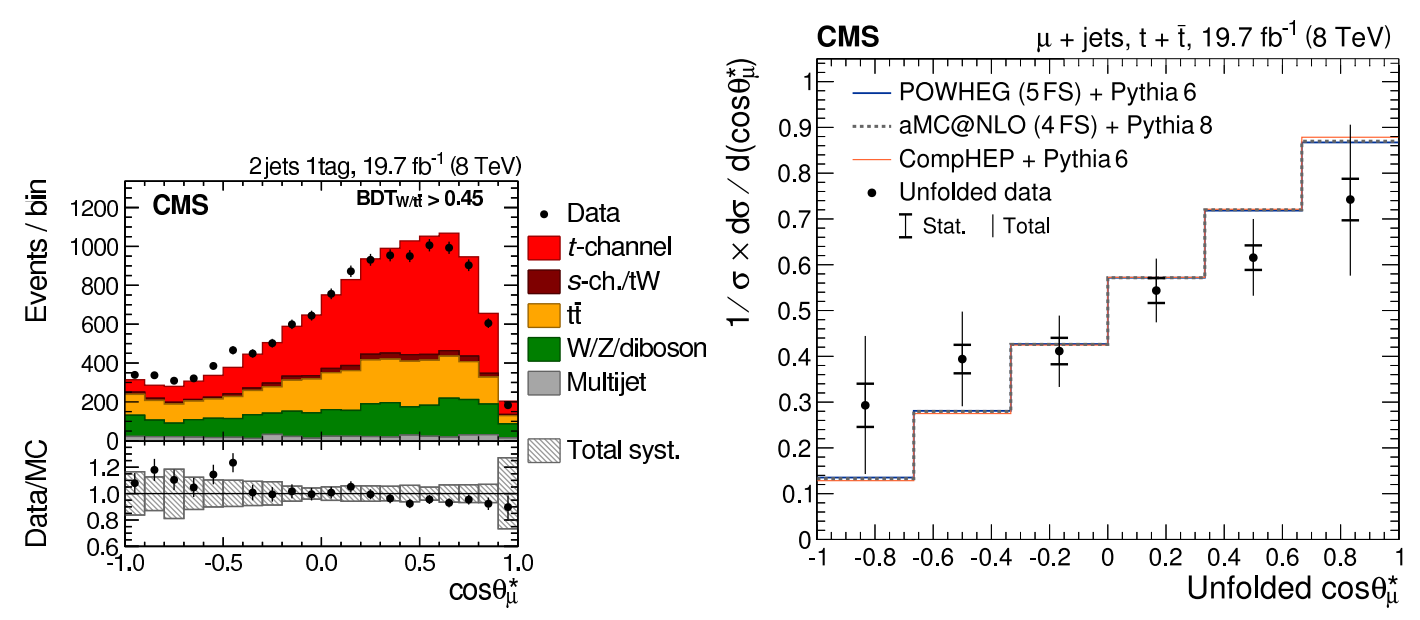

Figure 12: Distribution of $\cos \theta^{*}$ in the signal region (left) and the normalized differential cross section as a function of unfolded $\cos \theta^{*}$ for top quark and antiquark combined (right) [24].

$$
\mathscr{L}_{W t b}^{a n o m}=-\frac{g}{\sqrt{2}} \bar{b} \gamma^{\mu}\left(V_{L} P_{L}+V_{R} P_{R}\right) t W_{\mu}^{-}-\frac{g}{\sqrt{2}} \bar{b} \frac{i \sigma^{\mu v} q_{v}}{m_{W}}\left(g_{L} P_{L}+g_{R} P_{R}\right) t W_{\mu}^{-}+\text {h.c. }
$$

where $g$ is the weak coupling constant, $m_{W}$ and $q_{v}$ are the mass and the four-momentum of the W-boson, respectively. $P_{L, R} \equiv\left(1 \mp \gamma^{5}\right) / 2$ are the left- and right-handed projection operators, $\sigma^{\mu v}=i\left[\gamma^{\mu}, \gamma^{v}\right] / 2 . V_{L, R}$ and $g_{L, R}$ are the complex left- and right-handed vector and tensor couplings, respectively. In the SM, the only non-vanishing coupling at $\mathrm{LO}$ is $V_{L}=1$.

The triple differential decay rate of top quark is expressed in the form of parametrized spherical harmonics. The measured angular distributions are fitted with these parametrized functions, as shown in Fig. 14. Two-dimensional exclusion limits are extracted for the real and imaginary parts of the tensor anomalous coupling, $g_{R}$, and are presented in Fig. 15. The results are in agreement with the SM expectation for the vanishing $g_{R}$ at LO.

\subsection{FCNC with top quarks}

The processes with FCNC are forbidden at tree level in the SM by Glashow-Iliopoulos-Maiani (GIM) mechanism [26]. Such interactions are only possible at higher orders via loop induced processes and become highly suppressed. However, these processes could be significantly enhanced in various BSM scenarios. In FCNC with top quarks there is a transition of a top quark to an up or charm quark with an additional particle (gluon, $\gamma$, Z-boson, Higgs boson) involved in the interaction. FCNC production and decays with top quarks described with the following effective lagrangian:

$$
\begin{aligned}
\mathscr{L} & =\sum_{q=u, c}\left[\sqrt{2} g_{s} \frac{\kappa_{g q t}}{\Lambda} \bar{t} \sigma^{\mu v} T_{a}\left(f_{G q}^{L} P_{L}+f_{G q}^{R} P_{R}\right) q G_{\mu v}^{a}+\right. \\
& +\frac{g}{\sqrt{2} c_{W}} \frac{\kappa_{z q t}}{\Lambda} \bar{t} \sigma^{\mu v}\left(f_{Z q}^{L} P_{L}+f_{Z q}^{R} P_{R}\right) q Z_{\mu v}+\frac{g}{4 c_{W}} \xi_{z q t} \bar{t} \gamma^{\mu}\left(f_{Z q}^{L} P_{L}+f_{Z q}^{R} P_{R}\right) q Z_{\mu}-
\end{aligned}
$$




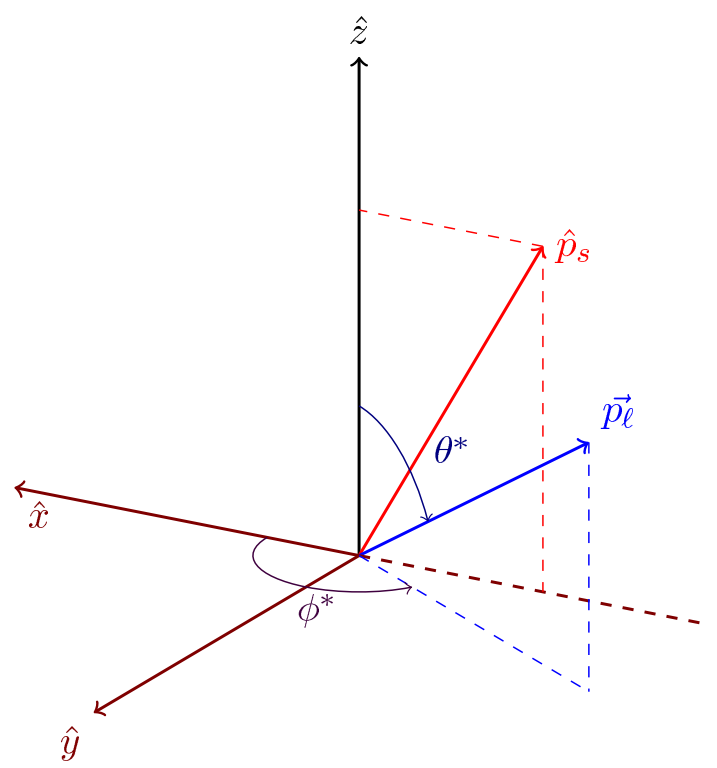

Figure 13: Definition of the coordinate system with $\hat{x}, \hat{y}$, and $\hat{z}$ defined as shown from the momentum directions of the $\mathrm{W}$ boson, $\hat{q}=\hat{z}$, and the spectator jet, $\hat{p}_{s}$, in the top quark rest frame. The angles $\theta^{*}$ and $\phi^{*}$ indicate the lepton direction $\hat{p}_{\ell}$ in this coordinate system [25].
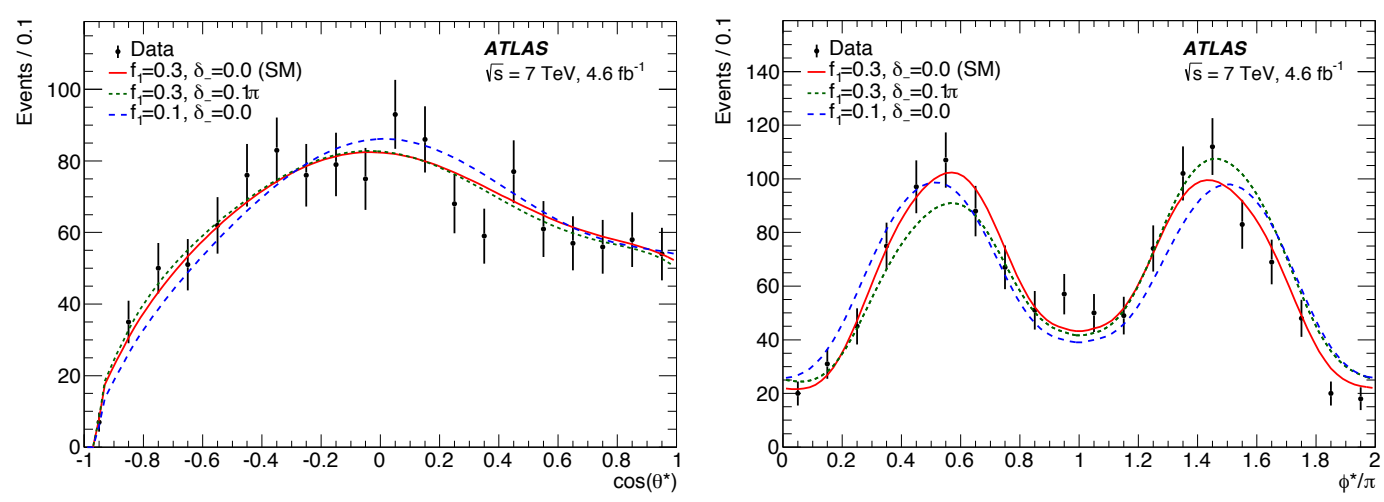

Figure 14: Projection of the double differential measurement into $\cos \theta^{*}$ (left) and $\phi^{*}$ (right) [25].

$$
\left.-e \frac{\kappa_{\gamma q t}}{\Lambda} \bar{t} \sigma^{\mu v}\left(f_{\gamma q}^{L} P_{L}+f_{\gamma q}^{R} P_{R}\right) q A_{\mu v}+\frac{g}{\sqrt{2}} \bar{t} \kappa_{H q t}\left(f_{H q}^{L} P_{L}+f_{H q}^{R} P_{R}\right) q H\right]+ \text { h.c. },
$$

where $\sigma^{\mu \nu}=\frac{1}{4}\left[\gamma^{\mu}, \gamma^{v}\right], P_{L}$ and $P_{R}$ are chirality projectors in spin space, $\kappa_{g q t}, \kappa_{z q t}, \kappa_{\gamma q t}$ and $\kappa_{H q t}$ are effective couplings with gluon, Z-boson, $\gamma$ and Higgs boson, respectively, $f_{G q}, f_{Z q}, f_{\gamma q}$ and $f_{H q}$ are chiral parameters, $\Lambda$ is the scale of the new physics.

An analysis done at ATLAS with $8 \mathrm{TeV}$ data looks for singly produced top quarks to probe the FCNC coupling with a gluon, $\kappa_{g q t}$ [27]. The final state considered in the analysis corresponds to the reconstructed products from the top quark leptonic decay. Neural network discriminant, shown in Fig. 16, is used to select signal events. The result limits on the coupling strength and branching ratios are presented in Fig. 17. Under assumption of only one non-vanishing FCNC coupling at 


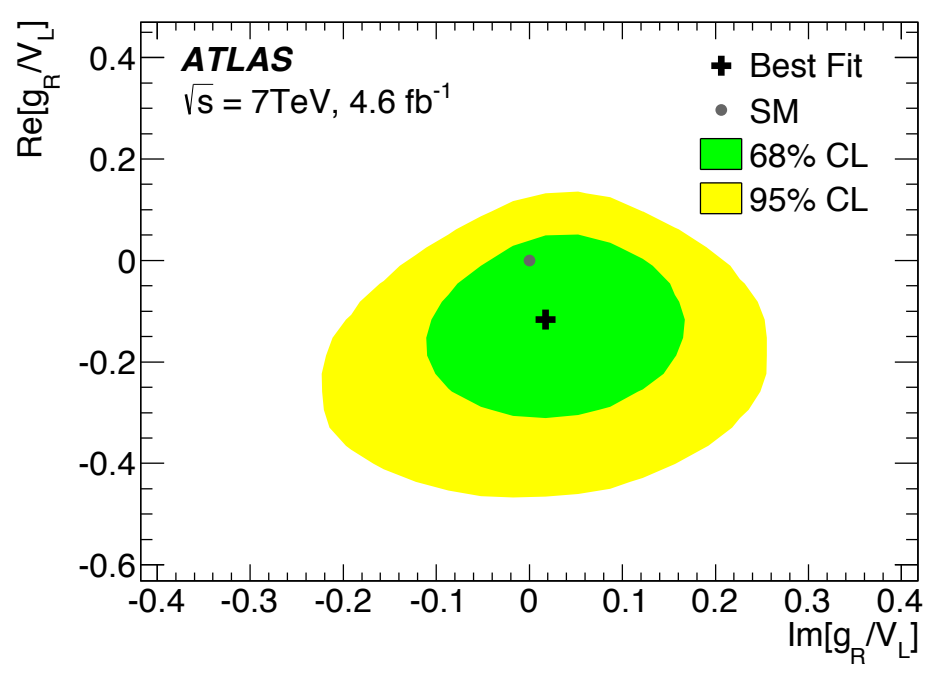

Figure 15: Two-dimensional exclusion limits at $95 \% \mathrm{CL}$ on the real and imaginary parts of $g_{R}$ anomalous coupling [25].

a time, the observed (expected) upper limits on the branching ratios at 95\% $\mathrm{CL}$ are $\mathscr{B}(t \rightarrow u g)<$ $0.0040(0.0035) \%$ and $\mathscr{B}(t \rightarrow c g)<0.020(0.018) \%$. These results represent the most stringent limits to date on these FCNC coupling and decay branching ratio.

Another analysis at CMS searches for single top production in association with a photon via FCNC interaction [28]. This is the only currently existing analysis at LHC that probes this type of FCNC coupling. The observed (expected) upper limits on the branching ratios at 95\% CL are $\mathscr{B}(t \rightarrow u \gamma)<0.01(0.02) \%$ and $\mathscr{B}(t \rightarrow c \gamma)<0.17(0.20) \%$. A summary for $\mathscr{B}(t \rightarrow q Z)$ and $\mathscr{B}(t \rightarrow q \gamma)$ limits is presented in Fig. 19 .

The latest experimental results from ATLAS and CMS on the limits for the branching ratios of FCNC decays of top quarks, compared to previous measurements obtained at Tevatron, HERA and LEP, shown in Figs. 20 and 21.

\section{Conclusion}

Processes with single top quarks allow one to perform tests of SM predictions and to search for new physics by probing anomalous couplings. Several new and refined results are available from LHC and Tevatron experiments. The measured cross sections for single top production processes are in a good agreement with theoretical predictions. Measurements of angular asymmetries due to the top quark polarization yield consistent results with the SM expectations. There is no sign for the new physics observed in the analysis of the top quark production rates and properties.

\section{References}

[1] D0 Collaboration, NIM A565 (2006) 463.

[2] CDF Collaboration, NIM A271 (1988) 387-403. 


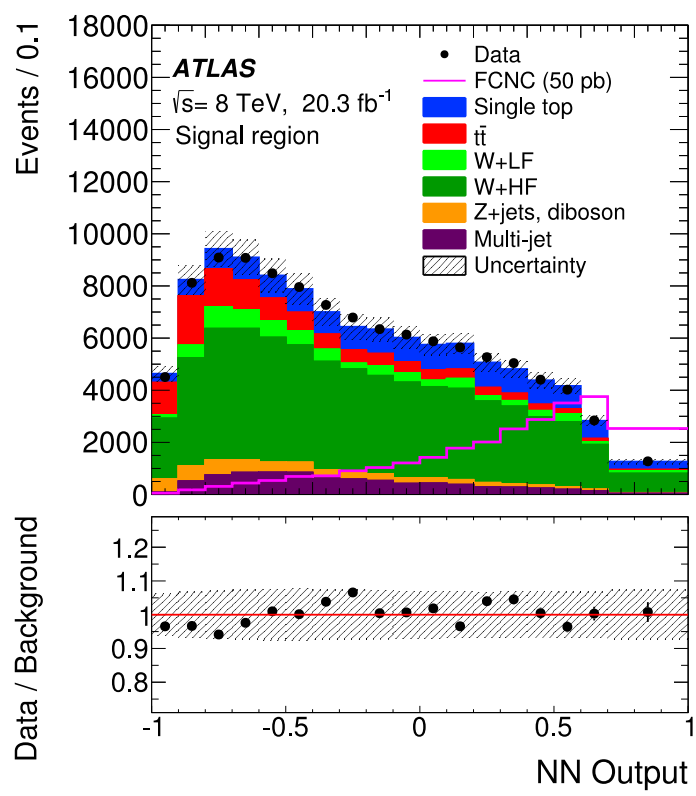

Figure 16: Neural network output distribution in the signal region [27].

[3] ATLAS Collaboration, JINST 3 (2008) S08003.

[4] CMS Collaboration, JINST 3 (2008) S08004.

[5] N. Kidonakis, Phys.Rev. D81 (2010) 054028.

[6] N. Kidonakis, Phys.Rev. D74 (2006) 114012.

[7] N. Kidonakis, Phys.Part.Nucl. 45 (2014) 714.

[8] J. M. Campbell, R. Frederix, F. Maltoni, and F. Tramontano, Phys.Rev.Lett. 102 (2009) 182003.

[9] N. Kidonakis, Phys.Rev. D82 (2010) 054018.

[10] CDF Collaboration, D0 Collaboration, Phys.Rev.Lett. 115 (2015) 152003.

[11] CDF Collaboration, D0 Collaboration, Phys.Rev.Lett. 112 (2014) 231803.

[12] CMS Collaboration, CMS PAS TOP-16-003.

[13] CMS Collaboration, CMS PAS TOP-15-004.

[14] ATLAS Collaboration, ATLAS-CONF-2015-079.

[15] CMS Collaboration, CMS PAS TOP-16-004.

[16] ATLAS Collaboration, Phys.Lett. B (2016) 228-246.

[17] ATLAS Collaboration, Phys.Lett. B740 (2015) 118.

[18] CMS Collaboration, arXiv:1603.02555.

[19] CMS Collaboration, Phys.Rev.Lett. 112 (2014) 231802.

[20] ATLAS Collaboration, JHEP 01 (2016) 064. 

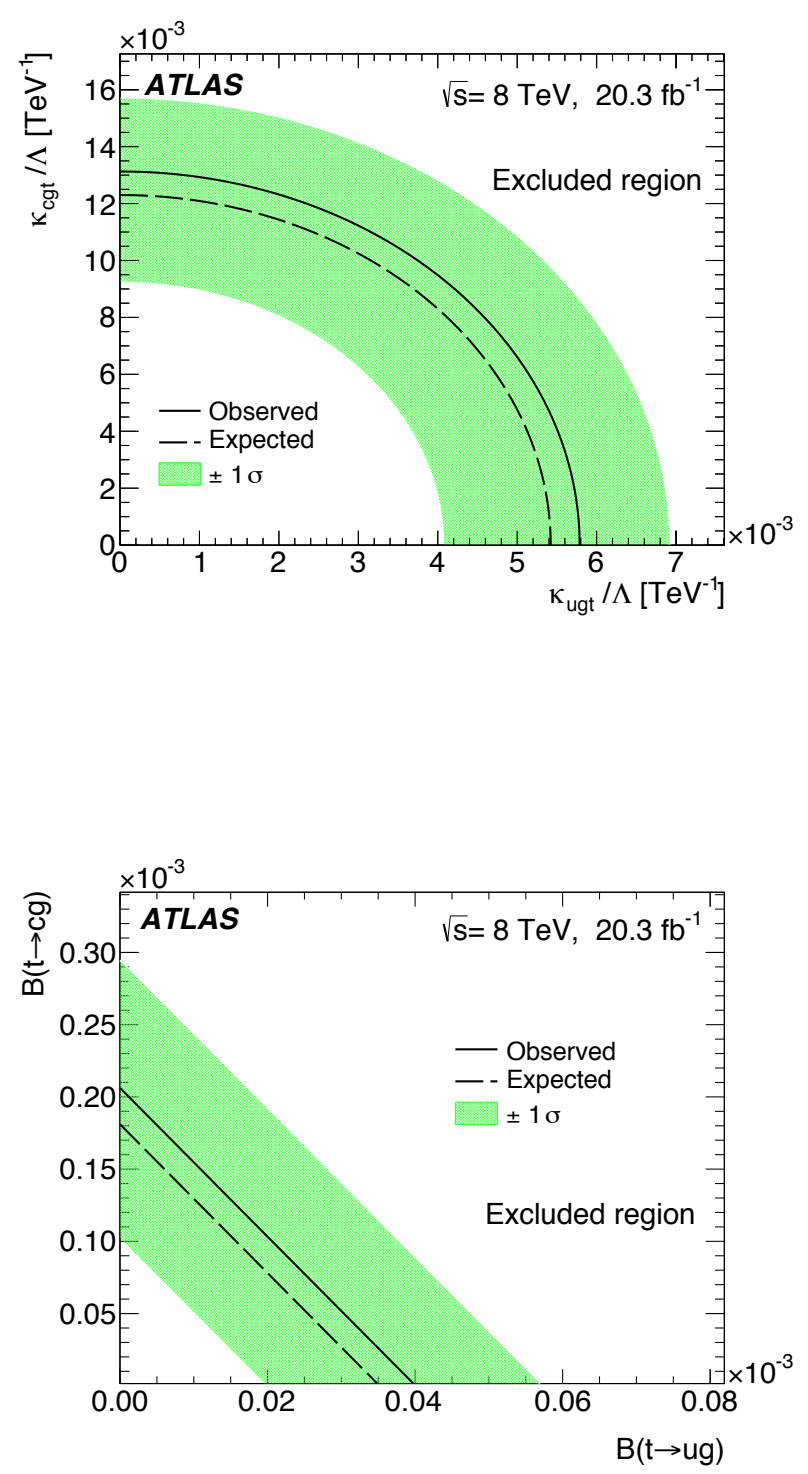

Figure 17: Upper limits on the coupling constants $\kappa_{g u t}$ and $\kappa_{g c t}($ top), and on the $\mathscr{B}(t \rightarrow u g)$ and $\mathscr{B}(t \rightarrow c g)$ (bottom) [27]. 


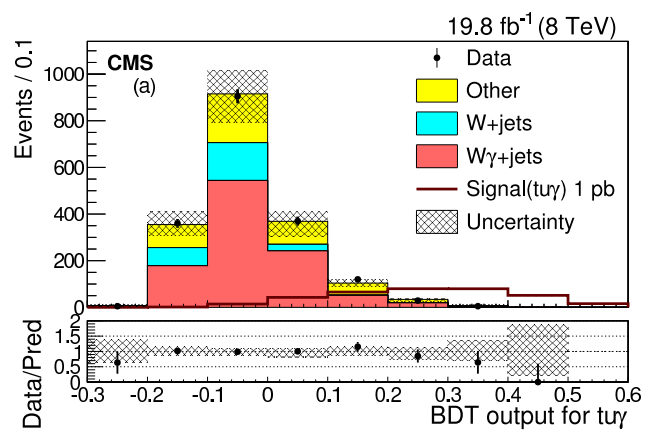

Figure 18: BDT output distribution in the signal region [28].

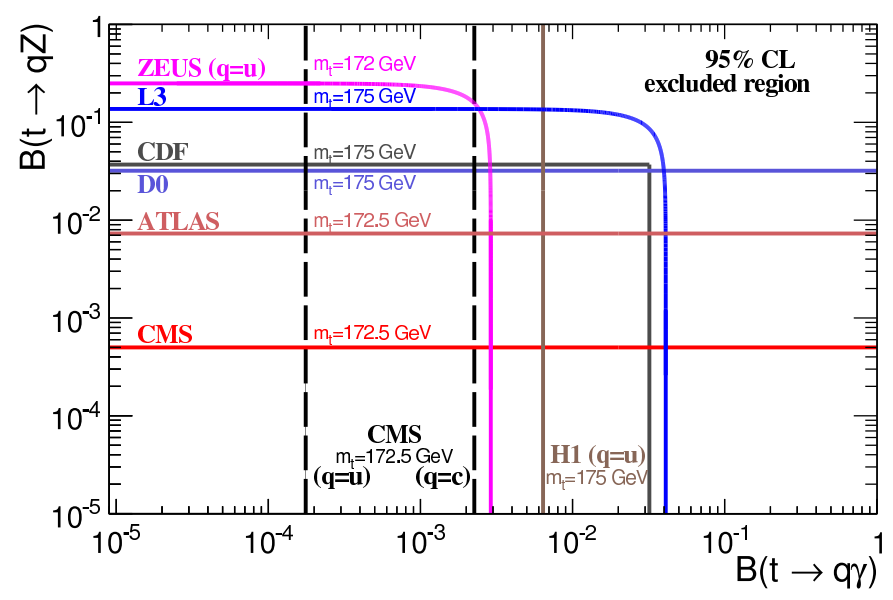

Figure 19: The measured 95\% CL upper limits on $\mathscr{B}(t \rightarrow q Z)$ versus $\mathscr{B}(t \rightarrow q \gamma)$ [28].

[21] ATLAS Collaboration, ATLAS-CONF-2016-023.

[22] CMS Collaboration, CMS PAS TOP-15-019.

[23] https://twiki.cern.ch/twiki/bin/view/LHCPhysics/LHCTopWGSummaryPlots.

[24] CMS Collaboration, JHEP 04 (2016) 073.

[25] ATLAS Collaboration, JHEP 04 (2016) 023.

[26] S.L. Glashow, J. Iliopoulos and L. Maiani, Phys.Rev. D2 (1970) 1285.

[27] ATLAS Collaboration, Eur.Phys.J. C (2016) 76:55.

[28] CMS Collaboration, JHEP 04 (2016) 035.

[29] https://atlas.web.cern.ch/Atlas/GROUPS/PHYSICS/CombinedSummaryPlots/TOP. 


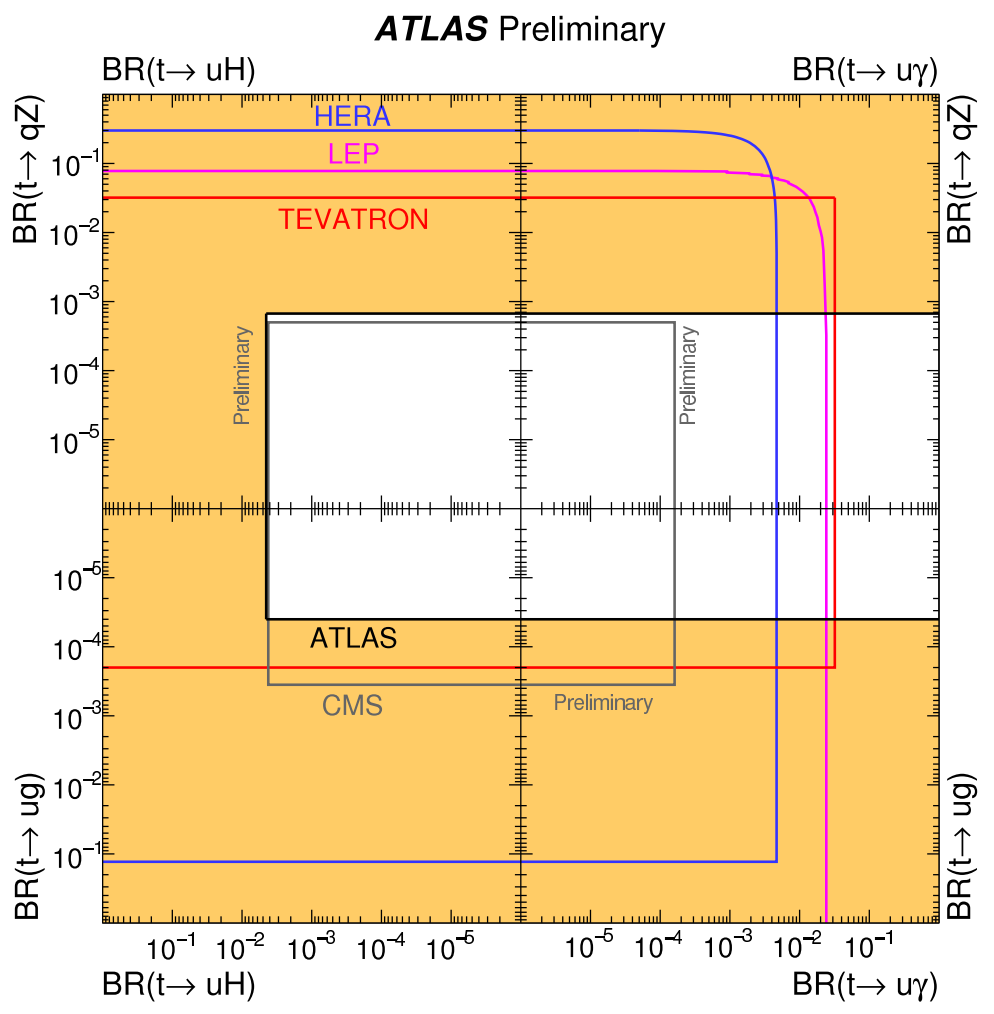

Figure 20: Summary of the latest $95 \%$ CL observed limits on the branching ratios of the top quark decays via FCNC to a charm quark and a neutral boson $\mathrm{t} \rightarrow \mathrm{uX}$ (X $=\mathrm{g}, \mathrm{Z}$-boson, $\gamma$ or Higgs boson) [29]. 


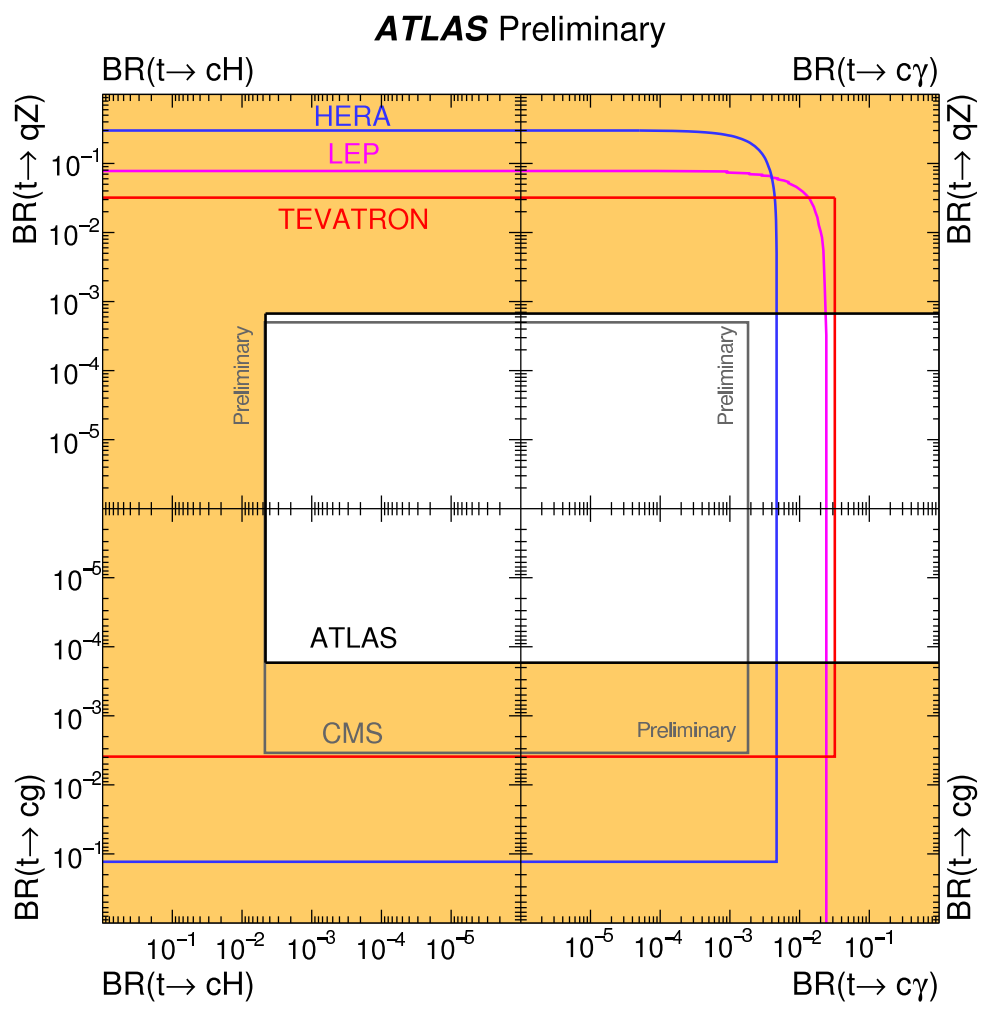

Figure 21: Summary of the latest $95 \%$ CL observed limits on the branching ratios of the top quark decays via FCNC to a charm quark and a neutral boson $\mathrm{t} \rightarrow \mathrm{cX}$ (X $=\mathrm{g}$, Z-boson, $\gamma$ or Higgs boson) [29]. 